\title{
OPEN Genotyping of methicillin resistant Staphylococcus aureus from the United Arab Emirates
}

\author{
Abiola Senok ${ }^{1 凶}$, Rania Nassar ${ }^{1,2}$, Handan Celiloglu ${ }^{1,3}$, Anju Nabi $^{4}$, Mubarak Alfaresi ${ }^{5}$, \\ Stefan Weber ${ }^{6}$, Irfan Rizvi ${ }^{3}$, Elke Müller ${ }^{7,8}$, Annett Reissig ${ }^{7,8}$, Darius Gawlik ${ }^{9,10}$, \\ Stefan Monecke ${ }^{7,8,11}$ \& Ralf Ehricht ${ }^{7,8,12}$
}

Reports from Arabian Gulf countries have demonstrated emergence of novel methicillin resistant Staphylococcus aureus (MRSA) strains. To address the lack of data from the United Arab Emirates (UAE), genetic characterisation of MRSA identified between December 2017 and August 2019 was conducted using DNA microarray-based assays. The 625 MRSA isolates studied were grouped into 23 clonal complexes (CCs) and assigned to 103 strains. CC5, CC6, CC22 and CC30 represented $54.2 \%(\mathrm{n} / \mathrm{N}=339 / 625)$ of isolates with other common CCs being CC1, CC8, CC772, CC361, CC80, CC88. Emergence of CC398 MRSA, CC5-MRSA-IV Sri Lanka Clone and ST5/ST225-MRSA-II, RhineHesse EMRSA/New York-Japan Clone in our setting was detected. Variants of pandemic CC8-MRSA[IVa + ACME I] (PVL+) USA300 were detected and majority of CC772 strains were CC772-MRSA-V (PVL+), "Bengal- Bay Clone". Novel MRSA strains identified include CC5-MRSA-V (edinA+), CC5-MRSA[VT + fusC], CC5-MRSA-IVa (tst1+), CC5-MRSA-[V/VT + cas + fusC + ccrA/B-1], CC8-MRSA-V/VT, CC22MRSA-[IV + fusC + ccrAA/(C)], CC45-MRSA-[IV + fusC + tir], CC80-MRSA-IVa, CC121-MRSA-V/VT, CC152MRSA-[V + fusC] (PVL+). Although several strains harboured SCC-borne fusidic acid resistance (fus $C$ ) $(n=181)$, erythromycin/clindamycin resistance $(\operatorname{ermC})(n=132)$ and gentamicin resistance (aacA-aphD) $(n=179)$ genes, none harboured vancomycin resistance genes while mupirocin resistance gene mupR $(n=2)$ and $c f r$ gene $(n=1)$ were rare. An extensive MRSA repertoire including CCs previously unreported in the region and novel strains which probably arose locally suggest an evolving MRSA landscape.

Methicillin resistant Staphylococcus aureus (MRSA) is an important cause of nosocomial infections worldwide and is associated with significant patient morbidity, mortality and healthcare costs. The epidemiology of MRSA has been dynamic and with an evolution towards increasing predominance of community associated MRSA lineages (CA-MRSA) as agents of hospital acquired MRSA (HA-MRSA) infections ${ }^{1,2}$. In recent years, emerging data on the molecular characterisation of $S$. aureus isolates in the hospital and community setting in countries of the Arabian Gulf region has contributed to changing our understanding of the diversity of isolates present ${ }^{3-9}$. In accordance with reports from other parts of the world, community associated MRSA (CA-MRSA) lineages have overtaken HA-MRSA lineages as aetiological agents also of nosocomial infections. Interestingly, MRSA isolates circulating in the region represent a wide clonal diversity $3,8,10-12$. Furthermore, emergence of novel and variant MRSA strains including those with novel SCCmec elements or with complex elements that include mecA as well as a gene associated with fusidic acid resistance $(f u s C)$ continue to be reported ${ }^{3,8,10}$.

${ }^{1}$ College of Medicine, Mohammed Bin Rashid University of Medicine and Health Sciences, P.O. Box 505055, Dubai, United Arab Emirates. ${ }^{2}$ Oral and Biomedical Sciences, School of Dentistry, Cardiff University, Cardiff, UK. ${ }^{3}$ Microbiology Department, Mediclinic City Hospital, Dubai Healthcare City, Dubai, United Arab Emirates. "Microbiology \& Infection Control Unit, Pathology Department, Rashid Hospital, Dubai Health Authority, Dubai, United Arab Emirates. ${ }^{5}$ College of Medicine, University of Sharjah, Sharjah, United Arab Emirates. ${ }^{6}$ Reference Laboratory for Infectious Diseases, Shaikh Khalifa Medical City, Abu Dhabi, United Arab Emirates. ${ }^{7}$ Leibniz Institute of Photonic Technology (IPHT), Jena, Germany. ${ }^{8}$ InfectoGnostics Research Campus Jena, Jena, Germany. ${ }^{9}$ PTC - Phage Technology Center GmbH, Bönen, Germany. ${ }^{10}$ Institute of Infectious Diseases and Infection Control, University Hospital, Jena, Germany. ${ }^{11}$ Institute for Medical Microbiology and Hygiene, Medical Faculty "Carl Gustav Carus", Technische Universität Dresden, Dresden, Germany. ${ }^{12}$ Institute of Physical Chemistry, Friedrich Schiller University Jena, Helmholtzweg 4, 07743 Jena, Germany. ${ }^{\boxplus}$ email: abiola.senok@ mbru.ac.ae 


\begin{tabular}{|l|l|}
\hline Clonal complex (CC) (\# of isolates in CC) & Number of strain assignments \\
\hline CC1 $(n=48)$ & 7 \\
\hline CC5 $(n=89)$ & 19 \\
\hline CC6 $(n=77)$ & 3 \\
\hline CC8 $(n=40)$ & 12 \\
\hline CC8 $/$ ST72 $(n=7)$ & 2 \\
\hline CC9 $(n=1)$ & 1 \\
\hline CC15 $(n=8)$ & 1 \\
\hline CC22 $(n=82)$ & 7 \\
\hline CC30 $(n=91)$ & 6 \\
\hline CC45 $(n=3)$ & 3 \\
\hline CC59 $(n=5)$ & 2 \\
\hline CC80 $(n=27)$ & 4 \\
\hline CC88 $(n=19)$ & 6 \\
\hline CC96 $(n=1)$ & 1 \\
\hline CC97 $(n=22)$ & 4 \\
\hline CC121 $(n=8)$ & 3 \\
\hline CC152 $(n=7)$ & 3 \\
\hline CC239 $(n=11)$ & 4 \\
\hline CC361 $(n=35)$ & 5 \\
\hline CC398 $(n=3)$ & 2 \\
\hline CC772 $(n=36)$ & 4 \\
\hline CC1153 $(n=4)$ & 2 \\
\hline CC2250 S. argenteus $(n=1)$ & 1 \\
\hline & \\
\hline & \\
\hline
\end{tabular}

Table 1. Clonal complex and strain assignments.

Although MRSA contributes to the burden of $S$. aureus infections in the United Arab Emirates (UAE), there remains a paucity of data on the molecular characterization of circulating strains. The UAE is a global tourist hub and home to a large expatriate population including those from regions from where there are hardly any data on MRSA prevalence and population structure. With this dynamic population, it is plausible that a diversity of MRSA clones can be found that includes highly transmissible pandemic clones as well as local strains from different regions from which tourists and expatriates might come to the UAE. Therefore, this study was carried out to determine the genotypes of MRSA isolates in the UAE and to differentiate closely related strains using a high-resolution typing method.

\section{Results}

CC diversity, virulence and antibiotic resistance genes. A total of 625 isolates obtained from wound/ pus swabs $(n=459)$, blood cultures $(n=95)$, respiratory sites (endotracheal aspirates/sputum) $(n=50)$ and urine $(n=21)$ were included in the study. Based on DNA microarray analysis, these 625 MRSA isolates were grouped into 23 clonal complexes (CCs) which were assigned to 102 strains (Table 1) with "strains" being defined based on their CC affiliation, toxin gene carriage and SCCmec type (see Supplementary Table S1 online for details of strain assignment). The CCs were identified across study sites and there was no clustering observed of any specific CC based on study site or type of clinical specimen. Four predominant CCs accounted for $54.2 \%(\mathrm{n} / \mathrm{N}=339 / 625)$ of isolates. These were CC30 $(\mathrm{n}=91 ; 14.6 \%)$, CC5 $(\mathrm{n}=89 ; 14.2 \%)$, CC22 $(\mathrm{n}=82 ; 13.1 \%)$ and CC6 $(\mathrm{n}=77 ; 12.3 \%)$ (Table 1). Other commonly identified CCs were CC1 $(n=48)$, CC8 $(n=40)$, CC772 $(n=36)$, CC361 $(n=35)$, CC80 $(n=27)$ and CC88 $(n=19)$. A majority of the strains belonged to CA-MRSA lineages harbouring SCCmec types IV and V (n/N=610/625; 97.6\%), (Supplementary Table S1 online). There was concordance between MRSA phenotypic and genotypic identification (data not shown).

We detected isolates harbouring SCC-borne fusidic acid resistance $(f u s C)$ gene $(\mathrm{n}=181)$, erythromycin/ clindamycin resistance $(\mathrm{erm} C)$ gene $(\mathrm{n}=132)$, and bifunctional enzyme gentamicin resistance $(a a c A-a p h D)$ $(\mathrm{n}=179)$. In addition, 25 isolates carried the fusidic acid resistance gene fus $B$ (also known as far 1 ). Carriage of the mupirocin resistance gene $\operatorname{mupR}(\mathrm{n}=2)$ and $23 \mathrm{~S}$ rRNA methyltransferase $c f r$ gene $(\mathrm{n}=1)$ was rare while none of our strains carried vancomycin / teicoplanin resistance genes (vanA, vanB, vanZ). The Panton Valentine leukocidin $(p v l)$ genes were present in $49 \%$ of all isolates studied while $13.8 \%$ were positive for the toxic shock syndrome (tst-1) gene. The prevalence rates of the antibiotic resistance and virulence genes are shown in Tables 2 and Table 3 respectively.

Emerging clonal complexes. Our findings document the first identification of CC398 MRSA in the Arabian Gulf region. The detection of $p v l$ genes and SCCmec typing using a second array revealed it to belong to the Asian, human adapted lineage rather than to the European livestock-associated one. Among CC5, we report the 


\begin{tabular}{|c|c|c|c|}
\hline \multicolumn{2}{|l|}{ Antibiotic resistance genes } & \multirow{2}{*}{\begin{tabular}{|l|} 
\# Positive $(\mathrm{N}=625)$ \\
625
\end{tabular}} & \multirow{2}{*}{ \% positive } \\
\hline Alternate penicillin binding protein 2, defining MRSA & mecA & & \\
\hline Mercury resistance operon & merA; merB & 0 & 0.0 \\
\hline SCCmec XI & mecC; blaZ-SCCmec XI & 0 & 0.0 \\
\hline Beta-lactamase operon & blaZ; blaI; blaR & 588 & 94.1 \\
\hline $\begin{array}{l}\text { rRNA adenine N-6-methyl-transferase, erythromycin/clindamycin resist- } \\
\text { ance }\end{array}$ & ermA & 15 & 2.4 \\
\hline Erythromycin/clindamycin resistance & ermB & 4 & 0.6 \\
\hline Erythromycin/clindamycin resistance & ermC & 132 & 21.1 \\
\hline Lincosamide Nucleotidyltransferase & $\operatorname{lin} A$ & 10 & 1.6 \\
\hline Energy-dependent efflux of erythromycin & $m s r A$ & 101 & 16.2 \\
\hline Acetyl-transferase inactivating streptogramin A & vatB & 0 & 0.0 \\
\hline ATP binding protein, Streptogramin A resistance & $\operatorname{vgaA}$ & 0 & 0.0 \\
\hline Bifunctional enzyme gentamicin resistance & aacA-aphD & 179 & 28.6 \\
\hline Amino-glycoside adenyl-transferase, tobramycin resistance & $a a d D$ & 47 & 7.5 \\
\hline 3'5'-Aminoglycoside phosphotransferase, neo-/kanamycin resistance & aphA3 & 138 & 22.1 \\
\hline Streptothricin acetyltransferase & sat & 129 & 20.6 \\
\hline Dihydrofolate reductase type 1 & $d f r A$ & 87 & 13.9 \\
\hline Fusidic acid resistance & fusB & 25 & 4.0 \\
\hline Hypothetical protein associated with fusidic acid resistance & Q6GD50 (fusC) & 181 & 29.0 \\
\hline Mupirocin resistance protein & mupR & 2 & 0.3 \\
\hline Tetracycline resistance & tet $\mathrm{K}$ & 85 & 13.6 \\
\hline Tetracycline resistance & tet $M$ & 18 & 2.9 \\
\hline Chloramphenicol acetyltransferase & cat & 5 & 0.8 \\
\hline 23S rRNA methyltransferase & $c f r$ & 1 & 0.2 \\
\hline Chloramphenicol/florfenicol exporter & fexA & 10 & 1.6 \\
\hline \multirow{2}{*}{ Metallothiol transferase } & fos $B$ & 403 & 64.5 \\
\hline & fosB-plasmid & 0 & 0.0 \\
\hline Quaternary ammonium compound resistance protein $\mathrm{A} / \mathrm{B}$ & qacA; qacC & 6 & 1.0 \\
\hline Transport-/efflux protein & tetEfflux & 539 & 86.2 \\
\hline Vancomycin resistance genes & van $A ; \operatorname{van} B$ & 0 & 0.0 \\
\hline Teicoplanin resistance gene from enterococci & $\operatorname{van} Z$ & 0 & 0.0 \\
\hline
\end{tabular}

Table 2. Detection of antibiotic resistance gene.

\begin{tabular}{|l|l|l|l|}
\hline Virulence genes & \# Positive (N=625) & \% positive \\
\hline Toxic shock syndrome toxin 1 & tst1 & 86 & 13.8 \\
\hline Panton Valentine leukocidin F/S component & lukF-PV; lukS-PV & 306 & 49.0 \\
\hline Staphylokinase & $s a k$ & 557 & 89.1 \\
\hline Chemotaxis-inhibiting protein & $c h p$ & 272 & 43.5 \\
\hline Staphylococcal. Complement inhibitor & $s c n$ & 597 & 95.5 \\
\hline Exfoliative toxin serotype A & $e t A$ & 3 & 0.5 \\
\hline Exfoliative toxin serotype B & $e t B$ & 1 & 0.2 \\
\hline Exfoliative toxin D & $e t D$ & 27 & 4.3 \\
\hline Epidermal cell differentiation inhibitor A & $e d i n A$ & 9 & 1.4 \\
\hline Epidermal cell differentiation inhibitor B & $e d i n B$ & 32 & 5.1 \\
\hline Epidermal cell differentiation inhibitor C & $e d i n C$ & 1 & 0.2 \\
\hline Arginine catabolic mobile element locus & ACME & 17 & 2.7 \\
\hline Staphylococcus aureus surface protein G & $\operatorname{sas} G$ & 512 & 81.9 \\
\hline
\end{tabular}

Table 3. Detection of virulence genes.

emergence of CC5-MRSA-IV Sri Lanka Clone and a second identification of ST5/ST225-MRSA-II, Rhine-Hesse EMRSA/New York-Japan Clone in the region (Supplementary Table S1 online). The CC5-MRSA-IV, Sri Lanka Clone is a CC5-MRSA carrying a SCCmec IVc element and $p v l$ genes as well as, variably, the enterotoxin genes sed, sej and ser. In CC8, different variants of the pandemic CC8-MRSA-[IVa + ACME I] (PVL+) USA300 strain were detected including one that lost arc genes and spe $G$ while retaining opp and copA2-SCC (copper resistance), 
as well as putative PVL-deletion mutants (Supplementary Table S1 online). For the first time in our region, we report identification of the ACME-negative/PVL-positive CC8-MRSA-[SCCmec IVc + Hg], which is a strain frequently described from Spain and Latin America (Supplementary Table S1 online). Another emerging strain was CC22-MRSA-IV harbouring $p v l$ and $t s t 1$ genes. A majority of the CC772 MRSA was assigned to CC772-MRSA$\mathrm{V}(\mathrm{PVL}+)$, "Bengal Bay Clone" while some of the CC772 isolates presented with other, unusual SCCmec variants.

Novel variant MRSA strains. We identified novel variant MRSA strains from 9 CCs, namely:

CC5 Four novel variant strains were identified, three of which carried SCCmec type V. All the strains carried regulatory and capsular genes agrII and cap5 respectively. Although all harboured the egc cluster enterotoxin genes, the edinA and tst-1 genes were found in single strains respectively. The CC5-MRSA-IVa $(t s t 1+)$ was the only one with SCCmec type IV and it uniquely harboured SCCmec IVa instead of usual IVc. Both CC5-MRSA-[VT $+f u s C]$ and CC5-MRSA-[V/VT $+c a s+f u s C+c c r A / B-1]$ carried the $f u s C$ gene. The CC5-MRSA-[VT + fusC] with SCCmec VT + fusC was identified as an unknown CC5/72 strain. A novel SCCmec element was exhibited by CC5-MRSA-[V/VT + cas $+f u s C+c c r A / B-1]$ which also carried the highest repertoire of antibiotic resistance genes among the novel strains (Table 3 ).

CC8 The novel variant strain in this CC was the CC8-MRSA-V/VT which uniquely harboured a SCCmec V sub-type (as in WIS; GenBank AB121219.1) and harboured the cassette chromosome recombinase genes $c c r C$ on the SCCmec element. No toxin associated genes were identified in this strain.

CC22 The CC22-MRSA-[IV + fus $C+c c r A A /(C)]$ had a new SCCmec element characterised by carriage of $\mathrm{SCC} m e c$ type IV with $c \mathrm{Cr} A A / C$ recombinase genes and $f u s C$ gene. In addition to egc cluster which is usually found in CC22 MRSA, this strain also harboured tst 1 gene.

CC45 The CC45-MRSA-[IV + fusC + tir] harboured the combination of $f u s C$ and tirS genes on the SCCmec element making is a novel CC45 variant strain.

CC80 CC80-MRSA nearly always harbour PVL and SCCmec IVc while lacking enterotoxin genes. However, this novel variant had SCCmec IVa, harboured enterotoxin genes (seb, sek, seq) and was negative for pvl genes.

CC121 Subtyping of the CC121 strains revealed the presence of a variant CC121-MRSA-VT strain with the SCCmec VT (GR1).

$\mathrm{CC} 152$ The CC152-MRSA- $[\mathrm{V}+f u s C](\mathrm{PVL}+)$ is the only novel variant strain harbouring the $p v l$ genes. The carriage of SCC $[\mathrm{mec} \mathrm{V}+f u s C]$ is novel in this CC.

CC361 One CC361 strain uniquely carried an SCCmec V / cas composite element. It also harboured tst1 gene and a complement of enterotoxin genes (sec, sel, egc cluster).

CC1153 Two PVL-positive CC1153-MRSA isolates were observed that presented with a mec complex B, ccrA/ $B 1$ recombinase genes and the fusC gene. Table 4 shows the genetic characterization of the novel strains.

\section{Discussion}

In recent years, emerging data globally and specifically in the Arabian Gulf region, have shown an evolving MRSA epidemiology with a shift to predominance of CA-MRSA lineages in nosocomial infections and an emergence of novel strains leading to an increased biological diversity of MRSA strains as well as to a greater diversity of SCCmec elements and variants thereof. In the UAE, MRSA contributes to the burden of infection and $30 \%$ of MRSA isolates identified at a tertiary care facility between 2011 and 2012 were CA-MRSA lineages ${ }^{13}$. Indeed, Sonnevend et al. also reported a trend of increasing CA-MRSA lineages between 2003 and 2008 at another tertiary care facility in the $\mathrm{UAE}^{14}$. However, data on the molecular characterization and strain assignments of circulating MRSA in the UAE are lacking. With the rapidly evolving changes reported in neighbouring countries and the dynamic population of the UAE, this study provides a much-needed snapshot of the genetic make-up of MRSA strains circulating in the UAE. Our findings demonstrate that an extensive MRSA repertoire of predominantly CA-MRSA lineage including CCs previously unreported in the region, plus rare and novel strains are present in the UAE. While the predominance of CC5, CC6, CC22 and CC30 is in accordance with reports from other countries in the region, identification of several pandemic MRSA strains and their variants such as CC8MRSA-[IVa + ACME I] (PVL+), USA300, CC22-MRSA-IV UK-EMRSA-15/Barnim EMRSA; CC30-MRSA-IV (PVL+), Southwest Pacific Clone and the HA-MRSA lineage CC239-MRSA-[mec III $+\mathrm{Cd} / \mathrm{Hg}+\mathrm{ccrC}$ ] is of concern in light of enhanced virulence, fitness and survivability of these strains. Furthermore, the first identification of CC398 MRSA heralds ominously the appearance of yet another previously unreported clonal complex in our region. The CC398 MRSA identified is the PVL-positive human variant of MRSA CC398 which is believed to have originated in South East Asia and is a frequent cause of infections in China and Vietnam ${ }^{15-17}$. In Europe, links to South East Asia were demonstrated in cases of infections and outbreaks associated with this CC398MRSA strain ${ }^{15,18}$. It is therefore highly likely that this strain was introduced to the UAE from South East Asia or even possibly via Europe.

CC5-MRSA are globally common ${ }^{19}$ with CC5-MRSA-IV (PVL+/edinA+), WA MRSA-121, CC5-MRSA$[\mathrm{IV}+f u s+c c r A B]$, "Maltese Clone" and CC5-MRSA-IV $(t s t 1+)$, being the prevalent strains in the Arabian Gulf region $^{3,4,6,20}$. Recently, the emergence of CC5-MRSA-VI strain including a novel variant which harboured the Staphylococcal TIR-protein binding protein gene (tirS) as additional payload on SCCmec was reported in Saudi Arabia ${ }^{8}$. Identification of eight CC5-MRSA-[VI $\left.+f u s C\right]$ including two with tirS in the current study suggests dissemination of this strain in the region. It has been postulated that the tirS gene confers enhanced bacterial survival and because it is located on a mobile genetic element in S. aureus, in this case an SCC element together with $f u s C$, horizontal gene transfer among MRSA strains is likely ${ }^{8,21}$. In addition to the CC5-MRSA we also 


\begin{tabular}{|c|c|c|c|c|c|c|}
\hline Clonal complex & Novel variants & $\begin{array}{l}\text { SCC mec-complex } \\
\text { associated genes }\end{array}$ & \begin{tabular}{|l|} 
Regulatory and capsule \\
genes
\end{tabular} & $\begin{array}{l}\text { Antibiotic resistance } \\
\text { genes }\end{array}$ & $\begin{array}{l}\text { Toxin associated } \\
\text { virulence genes }\end{array}$ & Other virulence genes \\
\hline \multirow{4}{*}{ CC5 } & $\begin{array}{l}\text { CC5-MRSA-V }(\text { edin } A+) \\
(\mathrm{n}=1)\end{array}$ & $\begin{array}{l}\text { ugpQ; mecA; ccrA-2; } \\
\text { ccrB-2 }\end{array}$ & agrII; cap5 & $\begin{array}{l}\text { blaZ; blaI; blaR; fosB; } \\
\text { tetEfflux }\end{array}$ & $e g c$ cluster & $\begin{array}{l}\text { hla; sak; chp; scn; edin } A \\
\text { icaA } A / C / D ; \text { clf } A / B ; \text { fnb } A / B\end{array}$ \\
\hline & $\begin{array}{l}\text { CC5-MRSA-[VT }+ \text { fusC] } \\
(\mathrm{n}=2)\end{array}$ & $\begin{array}{l}\text { ugpQ; mecA; Q6GD50 } \\
\text { (fusC); } c c r A A ; \text { ccrC }\end{array}$ & agrII; cap5 & $\begin{array}{l}\text { blaZ; blaI; blaR; fosB; } \\
\text { tetEfflux }\end{array}$ & seb; egc cluster & $\begin{array}{l}\text { hla; sak; scn; icaA/C/D; } \\
\text { clfA } B ; \text { fnbA/B }\end{array}$ \\
\hline & $\begin{array}{l}\text { CC5-MRSA-IVa (tst1+) } \\
(\mathrm{n}=1)\end{array}$ & $\begin{array}{l}\text { ugpQ; mecA; ccrA-2; } \\
\text { ccrB-2 }\end{array}$ & agrII; cap5 & $\begin{array}{l}\text { blaZ; blaI; blaR; fosB; } \\
\text { tetEfflux }\end{array}$ & tst1; sea; egc cluster & $\begin{array}{l}\text { hla; sak; chp; scn; } \\
\text { icaA/C/D;clfA/B;fnbA/B }\end{array}$ \\
\hline & $\begin{array}{l}\text { CC5-MRSA-[V/ } \\
\text { VT }+ \text { cas }+ \text { fus } C+c c r A / B-1] \\
(\mathrm{n}=1)\end{array}$ & $\begin{array}{l}\text { ugpQ; mecA; ccrA-1; } \\
\text { ccrB-1; ccAA; ccrC; } \\
\text { Q6GD50 (fusC); cas }\end{array}$ & agrII; cap5 & $\begin{array}{l}\text { blaZ; blaI; blaR; ermC; } \\
\text { tetK; tet } M \text {; cat; fexA; } \\
\text { fosB; tetEfflux }\end{array}$ & $\begin{array}{l}\text { sea; sed; sej; ser; egc } \\
\text { cluster }\end{array}$ & $\begin{array}{l}\text { hla; sak; scn; icaA/C/D; } \\
\text { clfA/B }\end{array}$ \\
\hline CC8 & $\begin{array}{l}\text { CC8-MRSA-V/VT } \\
(\mathrm{n}=1\end{array}$ & ugp Q; mecA; ccrC & agrI; cap 5 & $\begin{array}{l}\text { blaZ; blaI; blaR; far1; } \\
\text { fosB; tetEflux }\end{array}$ & - & $\begin{array}{l}\text { hla; icaA/C/D; clfA/B; } \\
\text { fnbA }\end{array}$ \\
\hline $\mathrm{CC} 22$ & $\begin{array}{l}\text { CC22-MRSA- } \\
{[\mathrm{IV}+\text { fus } C+c c r A A /(C)]} \\
(\mathrm{n}=1)\end{array}$ & $\begin{array}{l}\text { ugpQ; mecA; ccAA; ccrC; } \\
\text { Q6GD50 (fusC) }\end{array}$ & agrI; cap5 & blaZ; blaI; blaR & tst 1 ; egc cluster & $\begin{array}{l}\text { hla; sak; chp; scn; } \\
i c a A / C / D ; \text { clfA/B; cna; } \\
\text { fnbA/B }\end{array}$ \\
\hline CC45 & $\begin{array}{l}\text { CC45-MRSA- } \\
{[\mathrm{IV}+\text { fusC }+ \text { tir }]} \\
(\mathrm{n}=1)\end{array}$ & $\begin{array}{l}\text { ugpQ; mecA; ccrA-2; } \\
\text { ccrB-2; Q6GD50 (fusC); } \\
\text { tirS }\end{array}$ & agrI; cap 8 & tetEfflux & sek; egc cluster & $\begin{array}{l}\text { hla; sak; chp; scn; } \\
\text { icaA/C/D; clfA/B; fnbA }\end{array}$ \\
\hline $\mathrm{CC} 80$ & $\begin{array}{l}\text { CC80-MRSA-IVa } \\
(\mathrm{n}=1)\end{array}$ & $\begin{array}{l}\text { ugpQ; mecA; ccrA-2; } \\
\text { ccrB-2 }\end{array}$ & agrIII; cap 8 & $\begin{array}{l}\text { blaZ; blaI; blaR; ermC; } \\
\text { tetEfflux }\end{array}$ & seb; sek; seq; & $\begin{array}{l}\text { hla; sak; chp; scn; etD; } \\
\text { edinB; icaA/C/D; clfA/B; } \\
\text { fnbA/B }\end{array}$ \\
\hline CC121 & $\begin{array}{l}\text { CC121-MRSA-V/VT } \\
(\mathrm{n}=1)\end{array}$ & $\begin{array}{l}\text { ugpQ; mecA; ccrAA; } \\
\text { ccrC; Q6GD50 (fusC) }\end{array}$ & agrIV; cap 8 & $\begin{array}{l}\text { blaZ; blaI; blaR; aacA- } \\
\text { aphD; fosB; tetEfflux }\end{array}$ & egc cluster & $\begin{array}{l}\text { hla; sak; scn; etA; etB; } \\
\text { edinC; icaA/C/D; clfA } B ; \\
\text { fnbA/B }\end{array}$ \\
\hline CC152 & $\begin{array}{l}\text { CC152-MRSA- }[\mathrm{V}+f u s C] \\
(\mathrm{PVL}+) \\
(\mathrm{n}=1)\end{array}$ & $\begin{array}{l}\text { ugpQ; mecA; ccrAA; } \\
\text { ccrC; Q6GD50 (fusC) }\end{array}$ & agrI; agrIV; cap5 & $\begin{array}{l}\text { blaZ; blaI; blaR; ermC; } \\
\text { aacA-aphD; tetK; } \\
\text { tetEfflux }\end{array}$ & lukF-PV; lukS-PV & $\begin{array}{l}\text { hla; sak; scn; edinB; } \\
\text { icaA } / D ; \text { clf } A / B ; f n b A / B\end{array}$ \\
\hline CC361 & $\begin{array}{l}\text { CC361-MRSA-V (SCCmec } \\
\text { V/cas composite element) } \\
(\mathrm{n}=2)\end{array}$ & $\begin{array}{l}\text { ugpQ; mecA; } c c r A A ; \\
\text { ccrC; }\end{array}$ & agrI; cap8 & $\begin{array}{l}\text { blaZ; blaI; blaR; ermC; } \\
\text { aphA3; sat; tetK; fosB; } \\
\text { tetEfflux }\end{array}$ & tst 1 ; sec; sel; egc cluster & $\begin{array}{l}\text { hla; sak; chp; scn; } \\
\text { icaA/C/D; clfA/B; fnbA/B }\end{array}$ \\
\hline CC1153 & $\begin{array}{l}\text { CC1153-MRSA with SCC- } \\
\text { mec I+fusC (PVL+) } \\
(\mathrm{n}=2)\end{array}$ & $\begin{array}{l}\text { ugpQ; mecA; ccrA-1; } \\
\text { ccrB-1; Q6GD50 (fusC) }\end{array}$ & agrII; cap5 & $\begin{array}{l}\text { blaZ; blaI; blaR; ermC*; } \\
\text { tetEfflux }\end{array}$ & lukF-PV; lukS-PV & $\begin{array}{l}\text { hla; sak; scn; icaA/C/D; } \\
\text { clfA/B; fnbA/B }\end{array}$ \\
\hline
\end{tabular}

Table 4. Characterization of novel methicillin-resistant Staphylococcus aureus strains. ${ }^{*}$ Present in one isolate; agr accessory gene regulator; $c c r$ cassette chromosome recombinase gene; $u g p Q$ glycerophosphoryl diester phosphodi-esterase, associated with mecA; mecA alternate penicillin binding protein 2, defining MRSA; Q6GD50 ( fusC) hypothetical protein associated with fusidic acid resistance; cap, capsule gene; blaZ betalactamase; blaI beta lactamase repressor (inhibitor); blaR beta-lactamase regulatory protein; fosB Metallothiol transferase; tetEfflux transport-/efflux protein; tet $M / K$ tetracycline resistance markers; erm $C$; rRNA adenine $\mathrm{N}$-6-methyl-transferases causing erythromycin/clindamycin resistance; cat chloramphenicol acetyltransferase; fexA chloramphenicol/florfenicol exporter; aacA-aphD bifunctional enzyme gentamicin resistance; $a p h A 3$ 3'5'-aminoglycoside phosphotransferase, neo-/kanamycin resistance; sat streptothricin acetyltransferase; farl fusidic acid resistance; egc cluster: enterotoxins g,i,m,n,o,u; sea enterotoxin A; seb enterotoxin B; sec enterotoxin C; sed enterotoxin D; sej, enterotoxin J; sek enterotoxin K; sel enterotoxin L; seq enterotoxin Q; tst 1 toxic shock syndrome toxin 1 ; lukF-PV/lukS-PV, Panton Valentine leukocidin F/S component; hla, haemolysin alpha; sak staphylokinase; scn staphylococcal complement inhibitor; $c h p$ chemotaxis-inhibiting protein (CHIPS); edinA intercellular adhesion protein A/C/D; clfA/B clumping factor $\mathrm{A} / \mathrm{B} ; \mathrm{fn} b \mathrm{~A} / \mathrm{B}$ fibronectin-binding protein $\mathrm{A} / \mathrm{B}$.

identified two other strains with tirS namely a novel CC45 variant, CC45-MRSA-[IV + fusC + tir], and CC1-MRSA $[\mathrm{VT}+f u s C+t i r+c c r A B 1]$. These findings are supportive of the notion for on-going horizontal gene transfer among MRSA strains in our setting.

The occurrence of antibiotic resistance and virulence genes on MRSA SCCmec/fus genetic elements is suggestive of novel adaptive mechanisms ${ }^{22}$. A high consumption of fusidic acid in the population confers a selective advantage for the emergence and proliferation of strains carrying the fus $C$ gene $^{23}$. When $f u s C$ and $m e c A$ co-exist on the SCC element, fusidic acid use could promote MRSA in the community while beta-lactam use promotes fusidic acid resistance in the hospital. Hence, MRSA strains with SCCmec + SCCfus composite elements have a selective advantage in both the hospital and community settings. The high prevalence of MRSA strains with fusC gene plus presence of CC 80 MRSA (which usually harbour fusB) suggests on-going community misuse of fusidic acid as a driving factor for MRSA evolution in our setting. Therefore, future emergence of MRSA strains with increased bacterial fitness, resistance and virulence is possible, hence continued surveillance for early detection and responsible use of antibiotics is necessary.

Recently reported phylogenetic analysis of CC5 PVL-positive MRSA from four continents showed geographical clustering with the identification of the ST5-PVL-positive MRSA-IVc Sri Lanka clone ${ }^{24}$. Wider geographical spread of this clone was demonstrable with its identification in England and Australia where demonstrable links to Sri Lanka were not consistently established ${ }^{24}$. Our study documents the first report of CC5-MRSA-IV Sri Lanka Clone with two distinct variants based on carriage or absence of enterotoxin genes (sed, sej, ser) in 
our setting. The detection of CC5-MRSA-IV Sri Lanka Clone also underscores the need for continued surveillance to keep track of introduction of new MRSA strains into the population. While it is likely that this strain was introduced rather than arising de novo in the UAE, we were unable to establish direct travel links with Sri Lanka probably because this information was sought retrospectively and patients might have acquired the strains through contact with healthcare workers or carriers with epidemiological links. The ST5/ST225-MRSAII, Rhine-Hesse EMRSA/New York-Japan Clone identified in this study is a HA-MRSA lineage pandemic strain which has been reported in Europe, Asia, North America and Australia ${ }^{19,25}$. It has previously been identified in Kuwait and our findings demonstrate the second report in our region. Although variants of this MRSA strain harbouring arginine catabolic mobile element (ACME) have been reported, this was not evident in the strain found in this study ${ }^{25}$. Further identification of novel strains within CC5 including edinA + variant, and strains with new SCCmec elements including SCC $[\mathrm{VT}+\mathrm{f} u s C]$ and SCC-[V/VT $+c a s+f u s C+c c r A / B-1]$ are suggestive of ongoing genetic modification and recombination, among CC5-MRSA strains circulating in the UAE, or of importation from yet unidentified sources.

CC22 is a widespread clonal group which is prevalent in this region. Using high-resolution typing methods with SCCmec subtyping we had previously identified the regional presence of six distinct C22-MRSA-IV strains ${ }^{11}$ and recently an additional novel variant with SCCmec V/VT $+f u s C$ and $p v l$ was reported from Saudi Arabia ${ }^{8}$. In this study, these previously reported strains as well as the European pandemic strain CC22-MRSA-IV (UKEMRSA-15/Barnim EMRSA) were detected, indicative of strong links to Middle East, India and Europe for the evolution of CC22-MRSA in the UAE. In addition, our findings reflect on-going expansion of the diversity of CC22 MRSA strains with the identification of the previously undescribed CC22-MRSA-[IV $+f u s C+c c r A A /(C)$ which harboured a novel SCCmec element.

The CC772-MRSA-V (PVL+), Bengal Bay Clone is believed to have emerged in the Indian subcontinent from the same lineage as the widespread CC1 and CC5 strains ${ }^{26-28}$. In a recent report from Pakistan, a majority of the characterised MRSA strains belonged to this $\mathrm{CC}^{29}$. In Western Europe, infections with CC772-MRSA are commonly associated with previous travel or patient's ethnic origin from the Indian sub-continent ${ }^{26}$. Having been previously reported in Saudi Arabia, Oman and Kuwait, its identification in the UAE is not surprising giving the dynamic population movement with the Indian subcontinent $t^{4,9,30}$.

MRSA CC121, CC152, CC361, and CC1153 are considered to be emerging in our region as variant strains belonging to these CCs are continually reported. It is therefore not surprising that other novel variant strains identified in this study belonged to these CCs ${ }^{3,8}$. CC152-MRSA-V has been reported from Germany, Australia, Sweden, Switzerland, the Balkans, Saudi Arabia and Kuwait ${ }^{3,8,19,31}$. In accordance with reported literature, the CC152-MRSA-V identified in this study harbour the $p v l$ and $e d i n B$ genes, and lacked enterotoxin genes ${ }^{19}$. This strain carried erythromycin/clindamycin resistance erm $C$ gene which was not present in previously described strains $^{3,8}$.

The first identification of CC361 in Saudi Arabia was recently reported with detection of variant strains similar to those previously described in Kuwait indicative of regional spread ${ }^{3,8}$. Although CC361-MRSA had previously been described as a rare strain in the $\mathrm{UAE}^{19}$, and only one CC361-MRSA-[V/VT + fusC] was found as nasal colonizer in a recent study from UAE dental clinics ${ }^{32}$, the current findings suggest a wider occurrence of this CC. Similar to our findings, a recent report from Kuwait describing 102 novel MRSA variants identified 32 as CC361 strains ${ }^{3}$. With up to $5 \%(\mathrm{n} / \mathrm{N}=35 / 625)$ of our isolates belonging to CC361-MRSA inclusive of a novel strain with SCCmec V / cas composite element, it appears that we can no longer consider this as a rare MRSA lineage in our setting.

In conclusion, an extensive MRSA repertoire which includes CCs previously unreported in the region, plus pandemic, rare and novel variant strains are present in the UAE. Some strains detected occur in other countries, so a travel connection is possible. Others which have not been described elsewhere, probably evolved within the region. The lack of documentation of travel history for our patients is a limitation of this study as such information would have been helpful in mapping the travel connections. However, in a population as diverse as the UAE, it should be noted that importation of a strain from abroad could have multiple sources and prior travel by the patient represents only one piece of the puzzle. An on-going, sustained transmission of at least some of the "imported" clones might have already started within the UAE population and affecting individuals who previously did not travel. Nevertheless, our findings provide the first detailed information on the genotyping of MRSA isolates in the UAE, providing important baseline data for future surveillance work and whole genome sequencing of novel strains to help understand MRSA evolution and epidemiology in the UAE.

\section{Materials and methods}

Specimen collection and bacterial strains: MRSA isolates were identified between December 2017 and August 2019 at four diagnostic microbiology laboratories associated with secondary and tertiary care facilities, across three Emirates (Dubai, Abu Dhabi and Umm Al Quwain) in the UAE, with no routine inter-hospital transfer of patients. Apart from Abu Dhabi isolates which were obtained between December 2017 and August 2018, those from other Emirates were identified between December 2017 and August 2019. Only consecutive MRSA isolates associated with clinical infection were included with only one isolate per patient. There was no restriction on patient age for inclusion of isolates. However, all isolates obtained from specimens (nasal/axillary swabs) collected for MRSA screening were excluded. Identification of $S$. aureus and confirmation of methicillin resistance were performed using the Vitek automated platform (bioMérieux, Marcy-l'Étoile, France) in the diagnostic laboratory in accordance with manufacturer's instructions and Clinical and Laboratory Standards Institute guidelines ${ }^{33}$. Ethical approval was obtained from Mohammed Bin Rashid University, Dubai Scientific Research and Mediclinic Hospitals ethics committees (MBRU-IRB-2018-019; DSREC-05/2018_11 and CR/2018/42). As the study involved use of MRSA isolates already identified as part of routine diagnostic investigation, waiver of informed 
consent was granted by the above-mentioned ethics committees. All methods in the study were carried out in accordance with relevant guidelines and regulations. All MRSA isolates were stored at $-80^{\circ} \mathrm{C}$ pending molecular characterisation.

Molecular characterisation of isolates was carried out using the StaphyType DNA microarray (Abbott [Alere Technologies $\mathrm{GmbH}$ ], Jena, Germany). The previously described probes, primers, and procedures were used for the detection of species markers, virulence and resistance genes as well as for SCCmec subtyping ${ }^{19,34,35}$. Microarray images were taken and analysed using the dedicated reader and software (Alere Technologies). The analysis of presence or absence of target gene, assignment to clonal complex, sequence type and strains as well as SCCmec type was carried out as previously described ${ }^{19,34,35}$. Selected isolates were further characterised using a second array that facilitated assignment to SCCmec subtypes ${ }^{35}$.

\section{Data availability}

Data generated or analysed during this study are included in this published article.

Received: 29 May 2020; Accepted: 16 October 2020

Published online: 29 October 2020

\section{References}

1. Deurenberg, R. H. et al. The molecular evolution of methicillin-resistant Staphylococcus aureus. Clin. Microbiol. Infect. 13, $222-235$. https://doi.org/10.1111/j.1469-0691.2006.01573.x (2007).

2. Turner, N. A. et al. Methicillin-resistant Staphylococcus aureus: an overview of basic and clinical research. Nat. Rev. Microbiol. 17, 203-218. https://doi.org/10.1038/s41579-018-0147-4 (2019).

3. Boswihi, S. S. et al. Emerging variants of methicillin-resistant Staphylococcus aureus genotypes in Kuwait hospitals. PLoS ONE 13, e0195933. https://doi.org/10.1371/journal.pone.0195933 (2018).

4. Monecke, S. et al. Characterisation of MRSA strains isolated from patients in a hospital in Riyadh, Kingdom of Saudi Arabi. BMC Microbiol. 12, 146. https://doi.org/10.1186/1471-2180-12-146 (2012).

5. Sarkar, A. et al. Antimicrobial resistance and virulence markers in methicillin sensitive Staphylococcus aureus isolates associated with nasal colonization. Microb. Pathog. 93, 8-12. https://doi.org/10.1016/j.micpath.2016.01.008 (2016).

6. Senok, A., Ehricht, R., Monecke, S., Al-Saedan, R. \& Somily, A. Molecular characterization of methicillin-resistant Staphylococcus aureus in nosocomial infections in a tertiary-care facility: emergence of new clonal complexes in Saudi Arabia. New Microbes New Infect. 14, 13-18. https://doi.org/10.1016/j.nmni.2016.07.009 (2016).

7. Senok, A. et al. Genotyping of Staphylococcus aureus associated with nasal colonization among healthcare workers using DNA microarray. J. Infect. Dev. Ctries 12, 321-325. https://doi.org/10.3855/jidc.10328 (2018).

8. Senok, A. et al. Emergence of novel methicillin-resistant Staphylococcus aureus strains in a tertiary care facility in Riyadh, Saudi Arabia. Infect. Drug Resist. 12, 2739-2746. https://doi.org/10.2147/IDR.S218870 (2019).

9. Udo, E. E., Al-Lawati, B. A., Al-Muharmi, Z. \& Thukral, S. S. Genotyping of methicillin-resistant Staphylococcus aureus in the Sultan Qaboos University Hospital, Oman reveals the dominance of Panton-Valentine leucocidin-negative ST6-IV/t304 clone. New Microbes New Infect 2, 100-105. https://doi.org/10.1002/nmi2.47 (2014).

10. Senok, A. et al. Characterisation of a novel SCCmec VI element harbouring fusC in an emerging Staphylococcus aureus strain from the Arabian Gulf region. PLoS ONE 14, e0223985. https://doi.org/10.1371/journal.pone.0223985 (2019).

11. Senok, A. et al. Diversity of methicillin-resistant Staphylococcus aureus CC22-MRSA-IV from Saudi Arabia and the Gulf region. Int. J. Infect. Dis. 51, 31-35. https://doi.org/10.1016/j.ijid.2016.08.016 (2016).

12. Senok, A. C. et al. Investigating a rare methicillin-resistant Staphylococcus aureus strain: first description of genome sequencing and molecular characterization of CC15-MRSA. Infect. Drug Resist. 10, 307-315. https://doi.org/10.2147/IDR.S145394 (2017).

13. Dash, N., Panigrahi, D., Al Zarouni, M., Yassin, F. \& Al-Shamsi, M. Incidence of community-acquired methicillin-resistant Staphylococcus aureus carrying Pantone-Valentine leucocidin gene at a referral hospital in United Arab Emirates. APMIS 122, 341-346. https://doi.org/10.1111/apm.12150 (2014).

14. Sonnevend, A. et al. Change in meticillin-resistant Staphylococcus aureus clones at a tertiary care hospital in the United Arab Emirates over a 5-year period. J. Clin. Pathol. 65, 178-182. https://doi.org/10.1136/jclinpath-2011-200436 (2012).

15. Moller, J. K. et al. International travel as source of a hospital outbreak with an unusual meticillin-resistant Staphylococcus aureus clonal complex 398, Denmark, 2016. Euro Surveill. 24, 14. https://doi.org/10.2807/1560-7917.ES.2019.24.42.1800680 (2019).

16. Ngoc Thi, Vu. et al. Population structure of colonizing and invasive Staphylococcus aureus strains in northern Vietnam. J. Med. Microbiol. 65, 298-305. https://doi.org/10.1099/jmm.0.000220 (2016).

17. Yu, F. et al. Prevalence of Staphylococcus aureus carrying Panton-Valentine leukocidin genes among isolates from hospitalised patients in China. Clin. Microbiol. Infect. 14, 381-384. https://doi.org/10.1111/j.1469-0691.2007.01927.x (2008).

18. Nurjadi, D. et al. Import of community-associated, methicillin-resistant Staphylococcus aureus to Europe through skin and soft-tissue infection in intercontinental travellers, 2011-2016. Clin. Microbiol. Infect. 25, 739-746. https://doi.org/10.1016/j. cmi.2018.09.023 (2019).

19. Monecke, S. et al. A field guide to pandemic, epidemic and sporadic clones of methicillin-resistant Staphylococcus aureus. PLoS ONE 6, e17936. https://doi.org/10.1371/journal.pone.0017936 (2011).

20. El-Mahdy, T. S., El-Ahmady, M. \& Goering, R. V. Molecular characterization of methicillin-resistant Staphylococcus aureus isolated over a 2-year period in a Qatari hospital from multinational patients. Clin. Microbiol. Infect. 20, 169-173. https://doi. org/10.1111/1469-0691.12240 (2014).

21. Askarian, F. et al. A Staphylococcus aureus TIR domain protein virulence factor blocks TLR2-mediated NF-kappaB signaling. J. Innate Immun. 6, 485-498. https://doi.org/10.1159/000357618 (2014).

22. Ellington, M. J. et al. Emergent and evolving antimicrobial resistance cassettes in community-associated fusidic acid and meticillinresistant Staphylococcus aureus. Int. J. Antimicrob. Agents 45, 477-484. https://doi.org/10.1016/j.ijantimicag.2015.01.009 (2015).

23. Williamson, D. A. et al. High usage of topical fusidic acid and rapid clonal expansion of fusidic acid-resistant Staphylococcus aureus: a cautionary tale. Clin. Infect. Dis. 59, 1451-1454. https://doi.org/10.1093/cid/ciu658 (2014).

24. McTavish, S. M. et al. Genomic and epidemiological evidence of a dominant panton-valentine leucocidin-positive methicillin resistant Staphylococcus aureus lineage in Sri Lanka and presence among isolates from the United Kingdom and Australia. Front. Cell Infect. Microbiol. 9, 123. https://doi.org/10.3389/fcimb.2019.00123 (2019).

25. Schulte, B., Bierbaum, G., Pohl, K., Goerke, C. \& Wolz, C. Diversification of clonal complex 5 methicillin-resistant Staphylococcus aureus strains (Rhine-Hesse clone) within Germany. J. Clin. Microbiol. 51, 212-216. https://doi.org/10.1128/JCM.01967-12 (2013).

26. Blomfeldt, A. et al. Emerging multidrug-resistant Bengal Bay clone ST772-MRSA-V in Norway: molecular epidemiology 20042014. Eur. J. Clin. Microbiol. Infect. Dis. 36, 1911-1921. https://doi.org/10.1007/s10096-017-3014-8 (2017). 
27. Goering, R. V. et al. Molecular epidemiology of methicillin-resistant and methicillin-susceptible Staphylococcus aureus isolates from global clinical trials. J. Clin. Microbiol. 46, 2842-2847. https://doi.org/10.1128/JCM.00521-08 (2008).

28. Monecke, S. et al. Genome sequencing and molecular characterisation of Staphylococcus aureus ST772-MRSA-V, "Bengal Bay Clone”. BMC Res. Notes 6, 548. https://doi.org/10.1186/1756-0500-6-548 (2013).

29. Jamil, B. et al. Hospital-acquired methicillin-resistant Staphylococcus aureus (MRSA) from Pakistan: molecular characterisation by microarray technology. Eur. J. Clin. Microbiol. Infect. Dis. 37, 691-700. https://doi.org/10.1007/s10096-017-3161-y (2018).

30. Boswihi, S. S., Udo, E. E. \& Al-Sweih, N. Shifts in the clonal distribution of methicillin-resistant Staphylococcus aureus in Kuwait Hospitals: 1992-2010. PLoS ONE 11, e0162744. https://doi.org/10.1371/journal.pone.0162744 (2016).

31. Francois, P. et al. Methicillin-resistant Staphylococcus aureus, Geneva, Switzerland, 1993-2005. Emerg. Infect. Dis. 14, $304-307$. https://doi.org/10.3201/eid1402.070229 (2008).

32. Senok, A. et al. Molecular characterization of Staphylococcus aureus isolates associated with nasal colonization and environmental contamination in academic dental clinics. Microb. Drug Resist. https://doi.org/10.1089/mdr.2019.0318 (2020).

33. Clinical and Laboratory Standards Institute. Performance Standard for Antimicrobial Susceptibility Testing, M100 27th edn. (CLSI, Wayne, 2017).

34. Monecke, S. et al. Microarray-based genotyping of Staphylococcus aureus isolates from camels. Vet. Microbiol. 150, 309-314. https ://doi.org/10.1016/j.vetmic.2011.02.001 (2011).

35. Monecke, S. et al. Diversity of SCCmec elements in Staphylococcus aureus as observed in South-Eastern Germany. PLoS ONE 11, e0162654. https://doi.org/10.1371/journal.pone.0162654 (2016).

\section{Author contributions}

A.S., R.N., S.M., R.E. conceived and designed the study. H.C., A.N., M.A., S.W., I.R. contributed to sample collection and isolate identification. R.N., D.G., E.M., A.R., carried out the DNA microarrays. A.S., S.M., R.E. contributed to analysis and interpretation of DNA microarray data. All authors contributed to the data interpretation, preparation and critical revision of the manuscript. All authors approved the final manuscript and take responsibility for the published manuscript.

\section{Funding}

The project was funded by internal research grant from the College of Medicine, Mohammed Bin Rashid University of Medicine and Health Sciences, UAE (Ref\#: MBRU-CM-RG2018-07).

\section{Competing interests}

The authors declare no competing interests.

\section{Additional information}

Supplementary information is available for this paper at https://doi.org/10.1038/s41598-020-75565-w.

Correspondence and requests for materials should be addressed to A.S.

Reprints and permissions information is available at www.nature.com/reprints.

Publisher's note Springer Nature remains neutral with regard to jurisdictional claims in published maps and institutional affiliations.

(c) (i) Open Access This article is licensed under a Creative Commons Attribution 4.0 International License, which permits use, sharing, adaptation, distribution and reproduction in any medium or format, as long as you give appropriate credit to the original author(s) and the source, provide a link to the Creative Commons licence, and indicate if changes were made. The images or other third party material in this article are included in the article's Creative Commons licence, unless indicated otherwise in a credit line to the material. If material is not included in the article's Creative Commons licence and your intended use is not permitted by statutory regulation or exceeds the permitted use, you will need to obtain permission directly from the copyright holder. To view a copy of this licence, visit http://creativecommons.org/licenses/by/4.0/.

(C) The Author(s) 2020 